\title{
Growth and melting of the nematic phase: Sample thickness dependence of the Mullins-Sekerka instability
}

\author{
Jordi Ignés-Mullol* and Patrick Oswald ${ }^{\dagger}$ \\ Laboratoire de Physique, École Normale Supérieure de Lyon, 46 Allée d'Italie, 69364 Lyon Cedex 07, France
}

(Received 26 October 1999)

\begin{abstract}
In this article we report our systematic studies of the dependence on the sample thickness of the onset parameters of the instability of the nematic-isotropic interface during directional growth and melting, in homeotropic or planar anchoring.

PACS number(s): 61.30. $-\mathrm{v}, 68.10 .-\mathrm{m}, 81.10 . \mathrm{Fq}, 68.45 .-\mathrm{v}$
\end{abstract}

\section{INTRODUCTION}

The study of the onset of the cellular instability that arises at the nematic-isotropic liquid-crystal interface during directional growth allows for a simple observation of the linear regime $[1,2]$, something unpractical in experiments with binary alloys. Moreover, the fact that the solute diffusion coefficients in the nematic and in the isotropic phases are very similar, makes it possible to observe the cellular instability both during growth and during melting [3]. By analogy with the experiments of solidification of binary alloys, one can associate the observations at the nematic-isotropic interface with the Mullins-Sekerka instability of a planar front [4-6] in one dimension.

Earlier studies [1-3] showed that the cellular instability observed in the liquid-crystal interface is qualitatively well described by the Mullins-Sekerka theory. Based upon that model, one finds relationships between $v$ (the pulling velocity beyond which the planar front becomes unstable), $\lambda$ (the wavelength of the cellular instability that develops at a velocity $v$ ), and $G$ (the imposed temperature gradient) of the form $G=G(v), \quad \lambda=\lambda(v)$. These expressions correspond to the curves in the parameter space that separate regions where the planar front is stable from regions where it is unstable. These marginal stability curves can be evaluated numerically and, in the limit of small $v, \lambda$, and $G$, useful analytical approximations are obtained, namely

$$
\begin{gathered}
v=\frac{G}{\Delta T}\left(D_{I}+k D_{N}\right), \\
\lambda^{3}=(2 \pi)^{3} \frac{4 d_{0} T_{N I}}{\Delta T} \frac{D_{I}}{k\left(D_{I}+D_{N}\right)}\left(\frac{\Delta T}{G}\right)^{2} .
\end{gathered}
$$

Here, $\Delta T$ is the freezing range (or temperature range) at which nematic and isotropic phases coexist at the given impurity concentration; $k$ is the partition coefficient (ratio of the solute concentration in the nematic phase to the solute concentration in the isotropic phase at the interface); $T_{N I}$ is the equilibrium bulk phase transition temperature; $d_{0}=\gamma / L$ is

\footnotetext{
*Present address: Department of Chemistry, Tulane University, New Orleans, LA 70118.

†Electronic address: oswald@physique.ens-lyon.fr
}

the thermal capillary length whith $\gamma$ the surface tension and $L$ the latent heat per unit volume. $D_{I}$ and $D_{N}$ are the diffusion coefficients of the impurity in the isotropic and in the nematic phases, respectively.

The above equations correspond to the instability that arises during freezing of the isotropic phase, which we observe in samples with homeotropic anchoring $\left(D_{N}\right.$ is, therefore, $D_{\perp}$, since the impurity diffuses in the direction perpendicular to the average director field). Similar expressions can be obtained in the case when the cellular instability is observed during melting of the nematic phase by just swapping $D_{I}$ and $D_{N}$ and by replacing $k$ by $1 / k$. We observe the latter phenomenon when a planar anchoring is induced in the nematic phase [therefore, $D_{N}=D_{\|}\left(D_{\perp}\right)$ when the director is parallel (perpendicular) to the temperature gradient]. When the director makes an angle $\theta$ different from 0 or $\pi / 2$, the formulas are more complicated and can be found in Ref. [7].

It has been observed that the experiments of directional growth at the nematic-isotropic interface reproduce the phenomenology that characterizes the Mullins-Sekerka instability. Moreover, the shape of the marginal stability curves is not far from the predictions of that standard model. It has been observed, however, that comparison between the measurements in real experiments and the predictions from the theory lead to very unrealistic estimates for the different experimental parameters involved [8]. Putting it in a different way, the values of $v$ and $\lambda$ in growth experiments are sensibly larger than the expected ones [which are determined with the knowledge of the different physical parameters involved in Eqs. (1) from independent measurements]. Moreover, this departure increases dramatically with sample thickness.

The motivation for the work reported here is the fact that these discrepancies are not yet understood. The coupling of the elasticity of the nematic phase with the interfacial dynamics and with the diffusion of impurities has been proven to introduce too small a correction to account for the observed effects [9-11]. Another (probably crucial) effect that has yet to be introduced in a careful theoretical description is the three-dimensional shape of the interface. Caroli et al. [12] showed, in a very simplified analysis, that the presence of curvature in the shape of the meniscus across the sample thickness indeed modifies the threshold in a way compatible with the observations in usual plastic crystals [13]. On the other hand, thickness effects are quite different in nematics as we shall see later. 
The goal of the present article is to elaborate a systematic experimental analysis of the observed effects that the finite thickness of the samples has on the onset of the cellular instability. Our starting point is a comparison between our observations and the two-dimensional model [Eqs. (1)]. In order to do this, a good knowledge of the different physical parameters present in those equations is needed. In the Appendix we report the methods we have followed to measure most of those parameters. Moreover, we will look for trends in our data that can be used as a guideline for future models that attempt to understand this system and that, undoubtedly, will have to address the issue of three-dimensional effects.

In Sec. II, we give a brief description of the experimental procedure, which has been detailed elsewhere [2]. In Sec. III we present the main effects associated with the finite thickness of our samples that can be observed in the measurements. A careful analysis of the data reveals interesting trends in the dependence of the onset of the instability on both $G$ and $b$. A comparison between the two possible configurations of our system (planar or homeotropic anchoring) is presented in Sec. IV. Discussion and comments about our experimental findings follow in Sec. V. In the Appendix we describe the procedure followed to measure different relevant parameters of the system.

\section{EXPERIMENTAL PROCEDURE}

Our samples consist of $8 \mathrm{CB}$ (4-n-octylctanobiphenyl from Merck) with $5.5 \%$ wt. of liquid crystal I52 (4-ethyl-2-fluoro-4' -[2-(trans-4- $n$-pentylcyclohexyl)-ethyl]biphenyl from Merck) [14] acting as an impurity. The mixture is confined between two glass plates chemically and mechanically treated to induce either a homeotropic or a planar anchoring of the nematic phase [15]. To obtain homeotropic anchoring, the glass plates are dipped after cleaning in a $0.1 \%$ Merck ZLI 3124 silane solution dissolved in toluene and trichloromethane $(2: 1)$. They are then slowly removed from the solution. The glass plates must be dry when they come out of the solution, an important condition to succeed strong anchoring. For planar anchoring, the glass plates are dipped in a $5 \%$ dichlorodimethylsilane solution in toluene. They are then rinsed with toluene and rubbed in a single direction with a feltlike tissue. We have used cell spacings in the range $6 \mu \mathrm{m}-30 \mu \mathrm{m}$. A uniform gap between the two glass plates is achieved by means of either nickel wires or pieces of mylar film acting as spacers. The parallelism of the plates is optimized by maximizing the spacing between the interference fringes observed when light is reflected off the empty cells. Moreover, the actual value of the cell gap is controlled to within $\pm 1 \mu \mathrm{m}$ by focusing a microscope on the inner side of both glass plates. The samples are placed between two ovens kept at a constant and controlled temperature so that the nematic-isotropic phase transition temperature is achieved in the region between the ovens $[1,2]$. Calibration measurements, performed by sliding a dummy sample with a thermocouple embedded in the gap space, show that there is good linearity in the temperature distribution inside the gradient. It is important to minimize vertical temperature gradients that could arise if a temperature difference existed across the gap. The bottom plate of our samples is in direct contact with the ovens. The top plate of the samples, however, is not in direct contact with the top part of the ovens (to avoid compressing the sample, which could change its thickness and distort the director field), but there is a space of about $50 \mu \mathrm{m}$ in between, which is allowed in order to use the different sample thicknesses. We have observed that if this space is much larger, for instance of the order of $500 \mu \mathrm{m}$, a significant vertical temperature gradient is generated. The presence of this gradient has a very important effect in the measurements of the onset of instability since the critical velocity is divided by 2 . By contrast, the curve $\lambda_{c}$ vs $v_{c}$ remains the same. We have also observed that the measurements of the threshold velocity for the onset of nucleation are quite insensitive to the vertical temperature gradients (see the Appendix). The presence of a vertical temperature gradient seems to have an effect similar to reducing the thickness of the sample. In the data presented in this paper, we have been careful to minimize the presence of unknown vertical temperature gradients across the gap.

Our samples are displaced along the temperature gradient between the two ovens, with the $N-I$ interface perpendicular to that gradient, at a constant pulling velocity. Our choice of impurity is particularly well suited to this experiment since the densities of $8 \mathrm{CB}$ and 152 are very similar. Therefore, solute convection, which had been present in earlier experiments [2], should not play a significant role here. We have not observed any signs of convection even for the strongest temperature gradients we have used. On the other hand, the fact that the solute diffusion coefficient in the nematic and in the isotropic phases has the same order of magnitude allows for the observation of the cellular instabilities both during freezing of the isotropic phase and during melting of the nematic phase, provided the right experimental conditions are present. We have observed that imposing a homeotropic anchoring of the nematic molecules causes the nematic phase to wet the plates and allows the observation of the cellular instability during freezing. Conversely, a planar anchoring, which will favor wetting by the isotropic phase, allows for the observation of the cellular instability during melting. Another interesting feature of this mixture, which we have discovered during the experiments described here, is the fact that the anisotropy in the diffusion coefficient seems to be very small $\left(D_{\perp} \simeq D_{\|}\right)$.

\section{OBSERVED THICKNESS DEPENDENCE OF CRITICAL WAVELENGTH AND CRITICAL VELOCITY DURING GROWTH}

We have measured the threshold velocity for the onset of the cellular instability and the wavelength of the sinusoidal ripples at the onset for different sample thicknesses and for different temperature gradients. Data reported in this section correspond to experiments of directional growth of the nematic phase in homeotropic anchoring: samples are pulled towards the cold oven. Our measurements show a strong dependence of both $v$ and $\lambda$ on the sample thickness. Moreover, both quantities are measured to be larger than the ones predicted from the two-dimensional Mullins-Sekerka theory, with a departure that increases with sample thickness. We emphasize here that this behavior is different from what is observed in the usual plastic crystals, where the front is always more unstable than predicted by the theory for a pla- 
nar front [13]. A remarkable fact in nematics is that $\lambda$ appears to maintain the same functional dependence on $G$ as the one predicted by the planar model, unlike $v$, whose dependence is radically different. We have found ad hoc empirical models that contain the functional dependence observed among all the parameters. They should help in the characterization of the effect that the finite thickness of the samples has on the cellular instabilities.

\section{A. Critical wavelength}

Our analysis shows that the $\lambda$ vs $G$ data, for a given sample thickness, qualitatively agree with the functional dependence in Eq. (1b), namely, $\lambda \simeq G^{-2 / 3}$. We observe, however, that the ratio $\lambda / G^{-2 / 3}$ is thickness dependent, and that it increases with sample thickness. For all the samples we have measured, $\lambda$ is always larger than the value one would expect from Eq. (1b), and the departure increases with $b$ (see Fig. 1). Moreover, if we look at the change in $\lambda$ due to $b$ for a fixed temperature gradient (see Fig. 1) a linear trend is noticeable for the range of thicknesses we have used. A question that arises is the possibility to extrapolate our data to $b=0$, and whether the extrapolated wavelengths would agree with the predictions of the Mullins-Sekerka theory, given the observed trend that our measurements approach the model as $b$ decreases. No model is available to predict how the different parameters will depend on the sample thickness. Therefore, we will have to rely on our empirical results. The above observations suggest that $\lambda$ has a functional dependence on $G$ and on $b$, up to first order in $b$, of the form

$$
\lambda(b, G)=\left(A_{0}+A_{1} b\right) G^{-2 / 3} .
$$

According to this, $\lambda G^{2 / 3}$ is a function of $b$ only, and data obtained with different temperature gradients can be combined in order to obtain a better approximation to the constants $A_{0}$ and $A_{1}$. Fitting a straight line to $\lambda G^{2 / 3}$ with all values of $b$ and $G$ included, we get $A_{0}=(223 \pm 10)$ $\times 10^{-4} \mathrm{~cm}^{1 / 3} \mathrm{~K}^{2 / 3}$, and $A_{1}=16.6 \pm 1 \mathrm{~cm}^{-2 / 3} \mathrm{~K}^{2 / 3}$. On Fig. 1 we see that this approach generates an excellent fit to our data, not only when $\lambda$ vs $b$ is plotted, but also when Eq. (2) is plotted for each value of $b$ in the $\lambda$ vs $G$ plot. We conclude, therefore, that our data are consistent with a zerothickness extrapolation of $\lambda$ of the form $\lambda=(223 \pm 10)$ $\times 10^{-4} \mathrm{G}^{-2 / 3}$ (cgs units). If we assume, based on the above observations, that this zero-thickness extrapolation corresponds to the Mullins-Sekerka model, then we can compare the above value for $A_{0}$ to Eq. (1b). We have directly measured all the parameters present in that equation, except for $d_{0}$ (see the Appendix). We can, therefore, use the above result combined with Eq. (1b) to give an estimate for $d_{0}$, namely, $d_{0}=(3.9 \pm 0.8) \times 10^{-10} \mathrm{~cm}$, which is compatible with the values found in the literature [2]. It is interesting to observe that the above analysis gives a way of defining a thickness-dependent effective thermal capillary length $d_{0, \text { eff }}$, which would replace $d_{0}$ in Eq. (1b) in order to match the measured $\lambda$. One finds

$$
d_{0, \mathrm{eff}}=d_{0}\left(1+\frac{A_{1}}{A_{0}} b\right)^{3}
$$
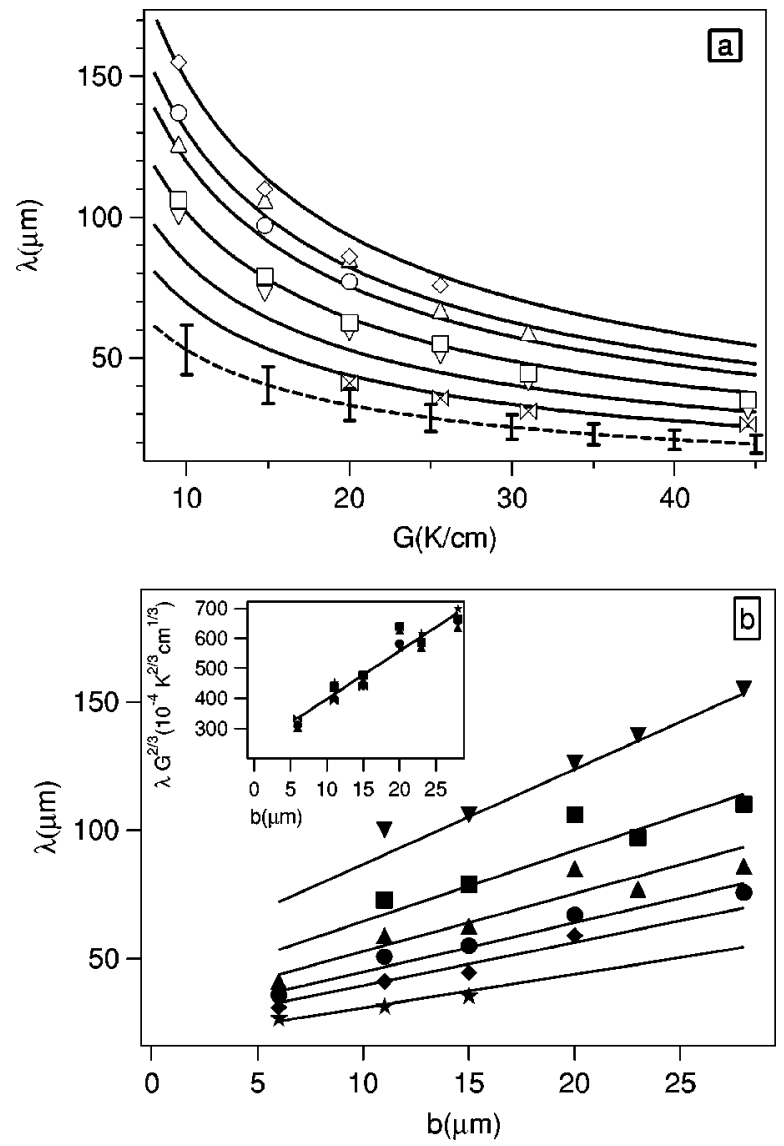

FIG. 1. Wavelength of the cellular modulations at the onset of the cellular instability during directional growth of a nematic sample. (a) Dependence on the applied temperature gradient for different sample thicknesses, namely, $b=6(\boldsymbol{M}), 11(\nabla), 15(\square)$, $20(\triangle), 23(\bigcirc)$, and $28(\diamond) \mu \mathrm{m}$. The dependence of $\lambda$ on $G$ is compatible with the functional dependence predicted by the Mullins-Sekerka theory ( $\lambda \simeq G^{-2 / 3}$, solid lines). The dashed line and the associated error bars show the interval where $\lambda$ vs $G$ should lie according to the two-dimensional model. (b) For the same data, we see that the wavelength increases roughly linearly with sample thickness, for a given value of $G$. Rescaling $\lambda$ with $G^{-2 / 3}$ collapses data taken with different $G$ (inset) and allows to extrapolate $\lambda$ at $b=0$ (see text). The temperature gradients are $G=9(\boldsymbol{\nabla})$, $15(\boldsymbol{\square}), 20(\boldsymbol{\Delta}), 26(\bullet), 31(\diamond)$, and $44(\star) \mathrm{K} / \mathrm{cm}$.

This parameter can also be thought of as the value for the thermal capillary length that one would estimate from measurements of $\lambda$ for samples of a given finite thickness.

\section{B. Critical velocity}

Similarly to what we observe with the measured values for $\lambda$, there is strong thickness dependence in $v$, and the measured values are always larger than those expected from the planar model. Unlike what we saw with $\lambda$, however, the functional behavior of $v$ vs $G$ is quite different from what the planar model predicts [see Eq. (1a)]. The observed $v$ grows much faster than linearly with $G$, something that becomes more notorious the larger $b$ is (see Fig. 2). At present, no theoretical work gives an explanation for this observed dependence. Similarly to what we have done above with $\lambda$, we can try to extrapolate the critical velocity to $b=0$, and compare that extrapolation to the predictions of the Mullins- 

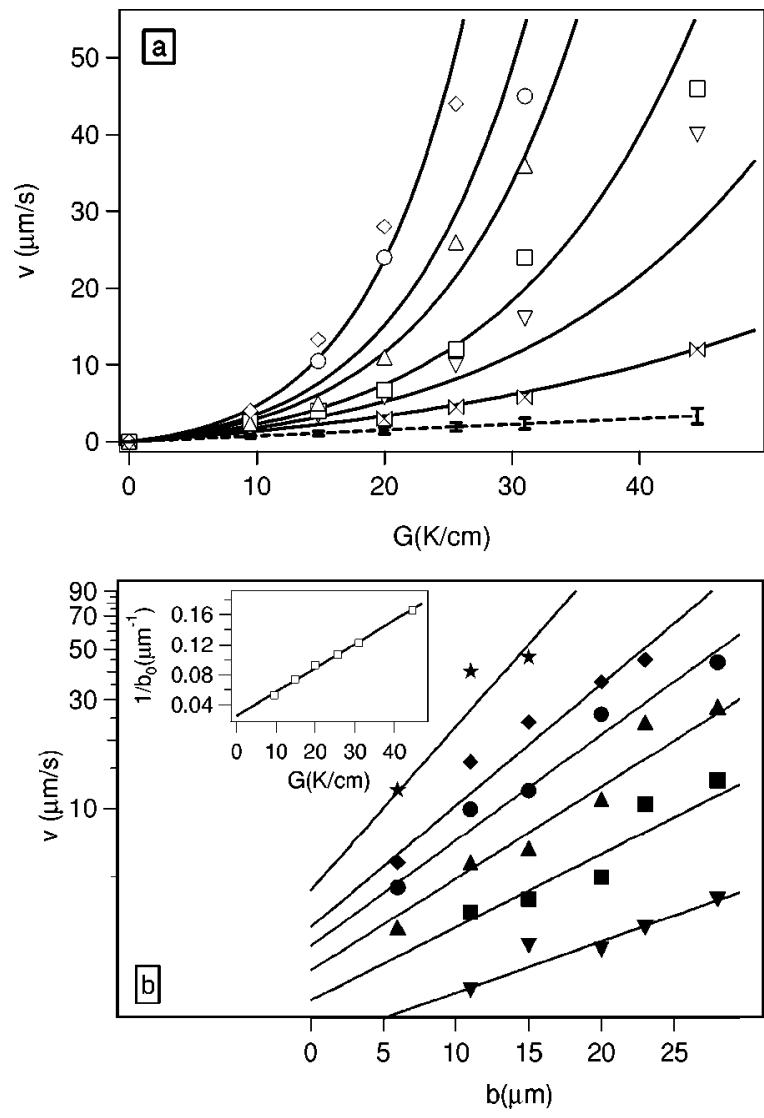

FIG. 2. For the same series of samples reported in Fig. 1 we measure the threshold velocity for the onset of the cellular instability. (a) Dependence on $G$. The solid lines correspond to a best fit to the data using an ansatz that includes both the dependence on $G$ and on $b$, as detailed below and in the text. The dashed line, and the corresponding error bars, are a conservative estimate of the range where $v$ vs $G$ should lie, according to the two-dimensional model and the measured physical parameters [see Eq. (1a)]. The sample thicknesses are $b=6(\bowtie), 11(\nabla), 15(\square), 20(\triangle), 23(\bigcirc)$, and $28(\diamond) \mu \mathrm{m}$. (b) For a given temperature gradient, $v$ increases roughly exponentially with $b$. The solid lines are fits to a relationship of the form $v=A G \exp \left[b / b_{0}(G)\right]$, where the parameter $A$, a constant, is obtained by simultaneously fitting all sets of data. The temperature gradients are $G=9(\boldsymbol{\nabla}), 15(\boldsymbol{\square}), 20(\boldsymbol{\Delta}), 26(\boldsymbol{\bullet})$, $31(\star)$, and $44(\star) \mathrm{K} / \mathrm{cm}$. In the inset, the inverse of the characteristic length scale $1 / b_{0}(G)$ is represented as a function of $G$. The solid line, intended to be a guide for the eye, is a linear fit to the data.

Sekerka theory. Since the departures from the planar model increase with $b$, it seems plausible to expect that we recover the expression in Eq. (1a) as $b$ approaches zero. A semilog plot of our data suggests that the increase of $v$ vs $b$ is close to exponential (see Fig. 2). A first approach at the $b=0$ extrapolation has been obtained by fitting an exponential to our data, $v=v_{0}(G) \exp \left[b / b_{0}(G)\right]$. We observe that the zerothickness extrapolation $v_{0}(G)$ is compatible with a linear increase with $G$. If this is the case, then a plausible ansatz for the functional dependence of our data is

$$
v=A G \exp \left(\frac{b}{b_{0}(G)}\right),
$$

where $A$ is a constant. In order to maximize the information that can be extracted from our data, we have performed a simultaneous fit of all data sets ( $G$ is constant for each data set) with this expression, obtaining a characteristic length $b_{0}(G)$ for each set. The result is a very reasonable fit to the data, and a $b_{0}(G)$ that varies smoothly with $G$ (see Fig. 2). In the range of $G$ measured, $1 / b_{0}(G)$ appears to increase linearly with $G$. If this is the case, then the gap dependence of $v$ is present even at small $G$, similarly to what we found with $\lambda$ [see Eq. (2)]. The factor $A$ can be directly compared with what would be expected from the Mullins-Sekerka theory [Eq. (1a)]. With the above ansatz, we obtain, for the slope of $v$ vs $G$ at $b=0, \quad A=(9.7 \pm 1) \times 10^{2} \mu \mathrm{m}^{2}$ $\mathrm{s}^{-1} \mathrm{~K}^{-1}$, while the slope of $v$ vs $G$ from Eq. (1a) is $\left(D_{I}\right.$ $\left.+k D_{N}\right) / \Delta T=(7.5 \pm 0.2) \times 10^{2} \mu \mathrm{m}^{2} \mathrm{~s}^{-1} \mathrm{~K}^{-1}, \quad$ obtained with the parameters measured for our system (see the Appendix). Our extrapolated velocities are noticeably larger than those expected from Eq. (1a). To further test the compatibility of the above ansatz with our data, we have plotted the expected $v$ vs $G$ relationship (4), for each $b$, using the values for $A$ and $b_{0}(G)$ obtained above. The result is a set of curves that closely match the $v$ vs $G$ data (see Fig. 2). Only a few points show a large scatter that may be attributable to experimental error. Each of the curves is particularly sensitive to inaccuracies in the knowledge of $b$ : variations of only a few percent in $b$ may result in over $20 \%$ variation in the ansatz curve for large $G$. The curve corresponding to the Mullins-Sekerka theory [Eq. (1a)] is shown for comparison with our data.

\section{COMPARISON WITH DIRECTIONAL MELTING}

In the preceding sections, we have presented experimental results obtained with samples where a homeotropic configuration was imposed on the nematic phase. As a result of that, a cellular instability resulted during growth, and nucleation due to superheating of the nematic phase occurred during melting. As we mentioned above, imposing an orientation of the director field such that it is parallel to the glass plates (planar orientation) we can observe the onset of the cellular instability upon directional melting of the nematic phase, while directional growth results in nucleation due to undercooling of the isotropic phase.

The coupling between diffusion anisotropy and front dynamics in directional growth experiments with liquid crystals has been addressed in the literature [7,10,17]. These studies showed that in planar anchoring, the cellular pattern must drift at the onset of instability in growth or in melting, provided that the director makes an angle different from 0 or $\pi / 2$ with respect to the interface. The fundamental reason is that the reflection symmetry about the growth axis is broken. The strength of this drift is given by the anisotropy of the diffusion coefficients in the nematic phase, $D_{\|}-D_{\perp}$. Another, more subtle effect, that is related to the elastic corrections in the Gibbs-Thomson relation, was discussed more recently by Misbah and Valence [10] and Bechhoefer and Langer [9]. The prediction is that elastic effects are completely negligible at the onset of instability, provided that a planar front is considered.

To test these ideas, we compared the onsets of the cellular instability in growth with homeotropic anchoring and in melting with planar anchoring. We found that the wave- 

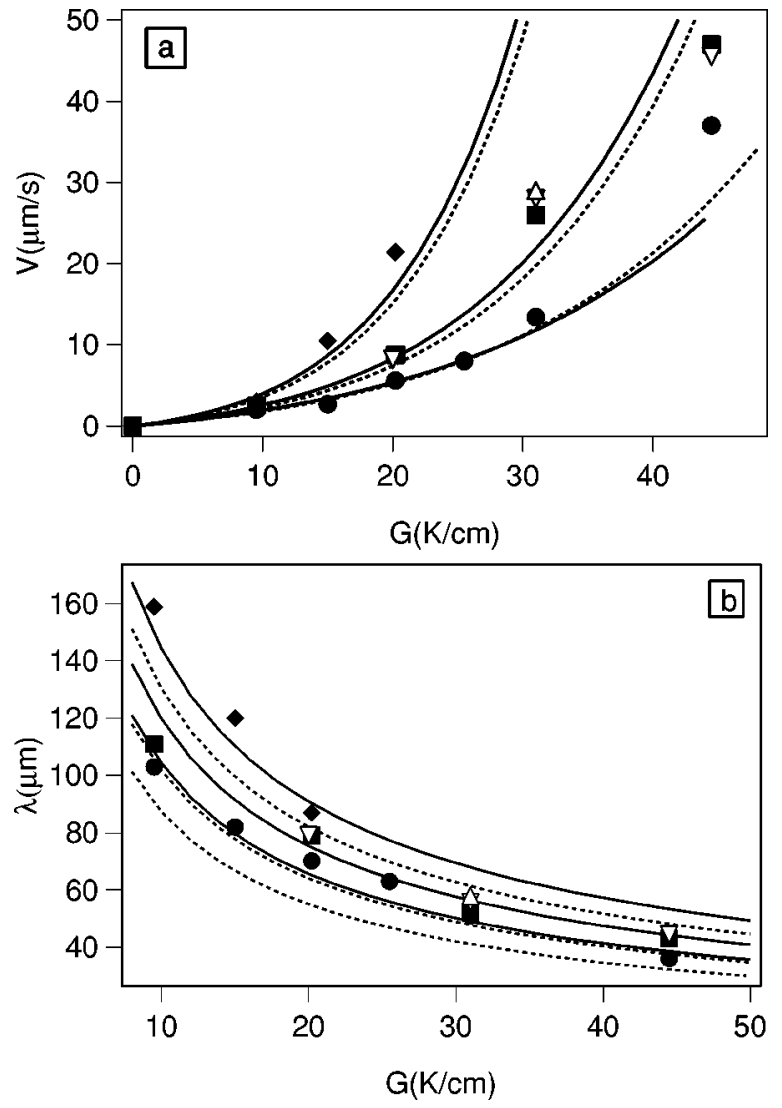

FIG. 3. (a) Velocity for the onset of the cellular instability upon melting the nematic phase for samples with planar anchoring with the director field parallel to $G$. (b) Wavelength of the sinusoidal instability at the onset. Solid symbols: the director field is parallel to the temperature gradient. The solid lines are the result of a simultaneous fit to the three data sets using the ansatz reported in Sec. III, Eqs. (2) and (4). Samples with $b=11$ (O), $b=15$ (ם), and $b$ $=21(\diamond) \mu \mathrm{m}$ are used. The dashed lines correspond to the curves that fit the data obtained for homeotropic anchoring with samples of similar thickness. For $b=15 \mu \mathrm{m}$, an extra series of measurements are reported for a planar sample oriented perpendicularly to $G(\nabla)$ and an extra data point corresponding to a planar sample oriented at $45^{\circ}$ from $G(\triangle)$.

lengths at the onset of instability are slightly different, whereas the threshold velocities are the same within our experimental errors. Moreover, we have changed the angle between the director field and the temperature gradient (or the interface). We have seen that neither the critical velocity, nor the wavelength of the cellular instabilities are affected significantly by this orientation (Fig. 3). On top of that, we have been unable to observe any cellular drift in any of the configurations we have used. In view of the results discussed above, the logical conclusion of these observations is that our system has a small anisotropy in the diffusion coefficient or, in other words, $D_{\|} \simeq D_{\perp}$.

Using now our measurements for melting of the planar nematic phase, we have performed an analysis similar to the one detailed in the previous sections. Melting of planar nematic samples shows similar qualitative results in what concerns the dependence of $v$ and $\lambda$ on $b$ and $G$. We find that the same ansatz proposed and tested for the instability during growth of nematic samples [Eqs. (2) and (4)] are equally valid here. We can, therefore, extract a similar set of param-

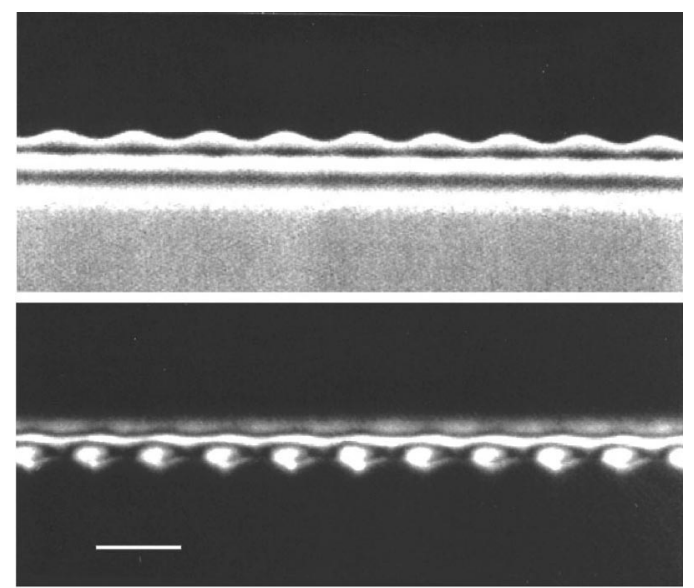

FIG. 4. Two snapshots of the nematic-isotropic interface at the onset of the instability for two samples with different anchoring geometry. Both observations have been performed in samples with $b=15 \mu \mathrm{m}, G=31 \mathrm{~K} / \mathrm{cm}$, and $v=28 \mu \mathrm{m} / \mathrm{s}$ (slightly above the threshold). Both pictures have been taken with the samples between crossed polarizers. In both cases, the nematic phase is underneath. Top: melting of a planar nematic sample. The polarizer is at $45^{\circ}$ of the interface. Bottom: growth of a homeotropic nematic phase. The polarizer is parallel to the interface. The line segment is $100 \mu \mathrm{m}$ long. Notice that the wavelength in the planar case is roughly $15 \%$ larger than in the homeotropic case (in the field of view we can fit 8.5 and 10 wavelengths, respectively).

eters from our data as we did above, and compare the results for both types of anchoring. In particular we can compare the $b=0$ extrapolation of $v$ and $\lambda$.

We see in Fig. 3 that the measurements of the critical velocity suggest that $v_{\mathrm{m}}$ and $v_{\mathrm{g}}$ are indistinguishable for all values of $b$ and $G$. The $b=0$ extrapolation is not an exception, and we get $A=(11 \pm 1) \times 10^{2} \mu \mathrm{m}^{2} \mathrm{~s}^{-1} \mathrm{~K}^{-1}$ [to be compared with $A=(9.7 \pm 1) \times 10^{2} \mu \mathrm{m}^{2} \mathrm{~s}^{-1} \mathrm{~K}^{-1}$, found above during growth]. The predictions of the MullinsSekerka theory for growth can be extended to melting by swapping $D_{I}$ and $D_{N}$, and changing $k$ by $1 / k$ in Eqs. (1). In this framework, the critical velocities at growth and at melting are related by $v_{\mathrm{g}}=k v_{\mathrm{m}}$, which corresponds to $v_{\mathrm{g}}$ $=1.06 v_{\mathrm{m}}$ in our system. Experimentally we find $v_{\mathrm{g}}=(0.9$ $\pm 0.2) v_{\mathrm{m}}$, which is not incompatible with the previous theoretical prediction, within experimental errors.

On the other hand, our measurements for $\lambda$ in the two configurations show that the anchoring imposed on the nematic phase has an important effect on the wavelength of the instabilities (see Figs. 3 and 4). From the predictions of the Mullins-Sekerka theory, one would expect the wavelengths at growth and at melting to be related by $\lambda_{\mathrm{g}}$ $=k^{-2 / 3}\left(D_{N} / D_{I}\right)^{-1 / 3} \lambda_{\mathrm{m}}$. For our system, this translates into $\lambda_{\mathrm{g}} \simeq 1.06 \lambda_{\mathrm{m}}$ (see the Appendix), which would make the two values indistinguishable given our experimental resolution. Our observations, however, show clearly that $\lambda_{\mathrm{g}}<\lambda_{\mathrm{m}}$ (see Figs. 4 and 5). In particular, if we compare the zerothickness extrapolations for $\lambda$ (which in both configurations are compatible with $\lambda \simeq G^{-2 / 3}$ ), we find the extrapolated values to be related by $\lambda_{\mathrm{g}} \simeq 0.7 \lambda_{\mathrm{m}}$ [we estimate $\lambda_{\mathrm{m}}(b=0)$ $=(3.4 \pm 5) \times 10^{-3} \mathrm{G}^{-2 / 3}$, in cgs units]. Clearly, one would expect to estimate the same value for the thermal capillary length, $d_{0}$, from measurements in both configurations, as 


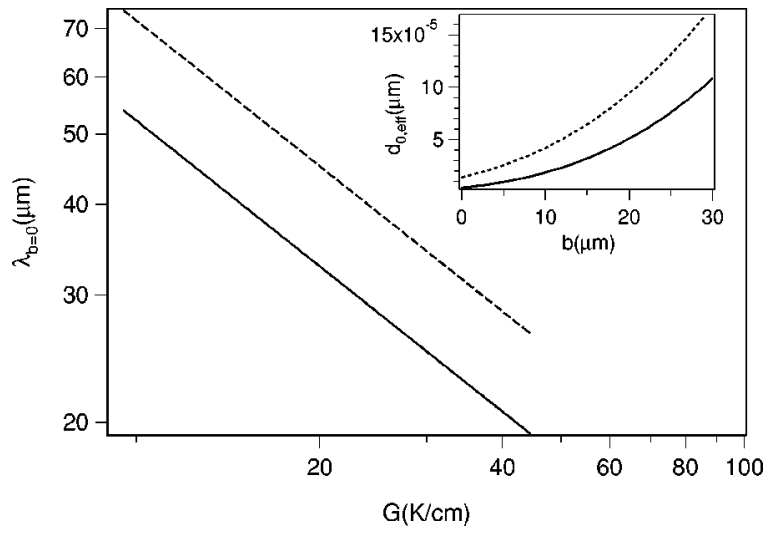

FIG. 5. Zero-thickness extrapolation of the wavelength of the cellular instabilities at the onset, as a function of $G$, for growth with a homeotropic sample (solid line) and melt with a planar sample (dashed line). In the inset, the effective, thickness-dependent thermal capillary length is extracted for measurements of growth (solid curve) and melting (dashed curve). Even at $b=0$ a substantial difference is observed in the determined capillary length in both configurations.

long as care is taken to use the right expressions for either case as noted above. In the planar configuration we get $d_{0}$ $=(13.8 \pm 4) \times 10^{-10} \mathrm{~cm}$, more than three times larger than the one found for the homeotropic configuration (see Sec. III A). The increasing departure between the wavelengths measured in either configuration with increasing sample thickness is illustrated by the parameter $d_{0, \text { eff }}$, defined in Eq. (3) (see Fig. 5).

\section{CONCLUDING REMARKS}

As we have reported in the previous sections, and as it has been reported before in the literature, the observed values for $v$ and $\lambda$ at the onset of the cellular instability approach those predicted by the Mullins-Sekerka instability of the planar front for small gaps or, at least, the observed departures from that theory are smaller for smaller gaps. We have more carefully analyzed this observation and found that, in fact, extrapolations of $v_{g}\left(v_{m}\right)$ and $\lambda_{g}\left(\lambda_{m}\right)$ to zero thickness are in qualitative agreement with the predictions of the MullinsSekerka model for a planar front. More precisely, $v_{g}$ and $v_{m}$ increase linearly with $G$ and approach their theoretical values to within $\pm 20 \%$ when $b \rightarrow 0$; the wavelengths $\lambda_{g}$ and $\lambda_{m}$ scale like $G^{-2 / 3}$ but their extrapolations to zero thickness in growth and in melting give different values for the capillary length $d_{0}$.

These results call for some comments. First, let us consider the events that take place as the pulling velocity approaches the threshold for instability. As reported before [1,3], a bright band forms between the isotropic liquid and the nematic phase, both in growth and in melting (Fig. 4). This band is due to the destabilization across the sample thickness of the meniscus that forms between the two phases. Experiments show that the width of this band saturates at about $l_{T}=\Delta T / G$ in thick samples $(b>10 \mu \mathrm{m})$, independently of $b$ and of the pulling velocity. We have observed that the meniscus seems to be systematically wider in melting than in growth (see Fig. 4). This effect could be an artifact due to the difficulty to optically detect the limits of the

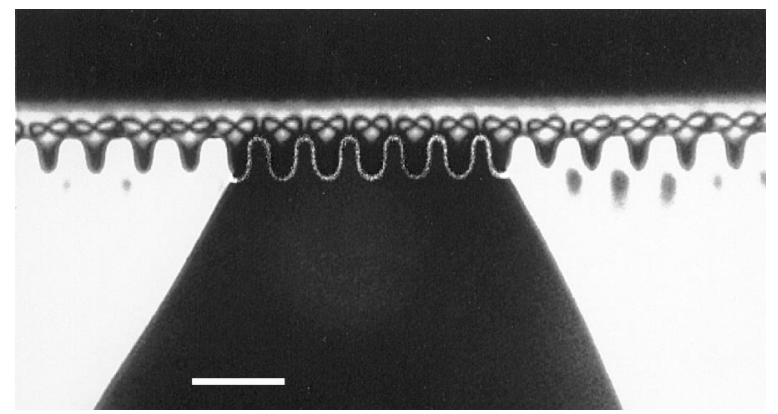

FIG. 6. The virtual disclination line that is attached to the front may detach during the growth of the homeotropic nematic. Triangles with a planar orientation inside form (white regions between crossed polarizers). The cell spacing (and the shape of the cells) are clearly the same in both regions. The apparent difference in the shape of cells in the homeotropic and in the planar regions is an artifact due to the saturation of the CCD video camera. The line segment is $100 \mu \mathrm{m}$ long. ( $b=15 \mu \mathrm{m}, G=31^{\circ} \mathrm{C} \mathrm{cm}^{-1}$, and $V$ $=30 \mu \mathrm{m} \mathrm{s}^{-1}$ ).

meniscus at the matching points with the glass plates. The critical velocities and wavelengths measured experimentally, therefore, correspond to the onset of destablization of the vertical part of the meniscus. Note that the thickness effects in nematics are completely different from those found in the usual plastic crystals, where the predictions of the planar model are approached at large thickness [13,19]. In other words, the thinner the samples are in usual systems, the more their behavior shifts from the linear theory for a planar front. This is in clear opposition to what we see in nematics. The main difference between solids and the nematic phase is that the meniscus have opposite curvatures.

The next point concerns the reason why, in nematics, the prediction of the Mullins-Sekerka instablity for a planar front are approached at zero thickness. One explanation could be that in this limit the amplitude of the deformation of the meniscus decreases below $\Delta T / G$, most probably due to the increasingly important capillary effect that tends to reduce the curvature of the meniscus. This capillarity-dominated regime should appear for gaps smaller than $\left(d_{0} T_{N I} / G\right)^{1 / 2}$, which is typically of the order of the micrometer. On the other hand, the front must remain curved at very small thicknesses, which could lead to some renormalization of the effective capillary length. In particular, the shape of the meniscus and its curvature in the vertical plane must strongly depend on the wetting angle at the triple point nematic isotropic glass. This angle may be different in growth and melting because our surface treatments are completely different in the two cases. This could explain why the two extrapolations of $d_{0}$ at zero thickness are different.

Another point concerns the virtual disclination line that is attached to the front [11]. This line sometimes detaches during growth of the nematic phase when a dust particle crosses the interface [1], forming a triangle with a planar orientation inside. We have observed that the critical velocities and the cell wavelengths are the same (within a few percent) in the homeotropic regions and inside the triangles (Fig. 6). In addition, we observed that the cells do not drift. These observations confirm that $D_{\|} \approx D_{\perp}$ in the nematic phase. This result obviously depends on the impurity chosen (I52 in the present experiment). Indeed, it has been reported in the lit- 
erature that when $\mathrm{C}_{2} \mathrm{Cl}_{6}$ (or more simply water) is chosen as an impurity, significant diffusion anisotropy results in the nematic phase: cells have then different wavelengths inside the triangles than in homeotropic regions $[1,3]$ and may drift even in homeotropic regions [16]. This cellular drift was first attributed to nonlinear effects [16] and was later reanalyzed in terms of mirror symmetry breaking due to the escape of the director field in the direction parallel to the interface, coupled with the anisotropy of the diffusion coefficients [18]. In our experiment, a similar three-dimensional structure of the director field is expected, but the absence of cellular drift at all thicknesses can again be interpreted as the result of the small anisotropy in $D_{N}$. We emphasize that elastic corrections to the Gibbs-Thomson relation are completely negligible in these experiments (because, otherwise the wavelength inside triangles would be different from the wavelength in homeotropic regions, which is not observed).

A final observation concerns the fact that the MullinsSekerka theory predicts that the linear relationships in Eqs. (1) can be expected for $G \ll G_{\max }$, where $G_{\max }$ is the temperature gradient beyond which the front is always stable. That model gives $G_{\max } \simeq(\Delta T)^{2} /\left(d_{0} T_{c}\right)$. With the parameters from our system (see the Appendix), one estimates $G_{\max }$ $\simeq 10^{4} \mathrm{~K} / \mathrm{cm}$, orders of magnitude higher than the gradients we have used. Earlier works showed that the actual value for $G_{\max }$ measured experimentally is much smaller than the theoretically predicted one. From our observations, it is to be expected that $G_{\max }$ is also thickness dependent. An interesting possibility, suggested by our results with the measurements of $\lambda$ vs $G$, is that the above expression for $G_{\max }$ might hold replacing $d_{0}$ by the effective value, $d_{0 \text {,eff }}$ that we can extract from our analysis.

\section{ACKNOWLEDGMENTS}

This work was supported by the European Research Network through Contract No. FMRX-CT96-0085.

\section{APPENDIX: VALUES FOR THE RELEVANT PHYSICAL PARAMETERS}

The nature of the discrepancies between the observed onset of the cellular instability and the two-dimensional Mullins-Sekerka theory can only be quantified if sufficient knowledge of the relevant physical parameters of the system is achieved. Our particular choice of impurity, I52, will have an influence mostly on the value of the diffusion coefficients in $8 \mathrm{CB}$ and on the details of the phase diagram, particularly in the slopes of the liquidus and solidus lines. We have placed great effort on measuring these parameters directly in our system, and we have relied on the values published in the literature for other, less impurity sensitive parameters.

By carefully measuring the temperature range at which the nematic and isotropic phases coexist for a given impurity concentration, we have measured the liquidus and the solidus lines. From there, $k=1.06 \pm 0.04$ and $m=0.58 \pm 0.03 \mathrm{~K} / \mathrm{wt} . \%$ are obtained. Particular care is taken to measure the freezing range for the impurity concentration reported in this paper, obtaining $\Delta T=0.21 \pm 0.01 \mathrm{~K}$ for $c=5.5 \%$ in weight $\left(T_{N I}\right.$ $\simeq 316 \mathrm{~K}$ at this impurity concentration).

A more delicate task is the accurate measurement of the

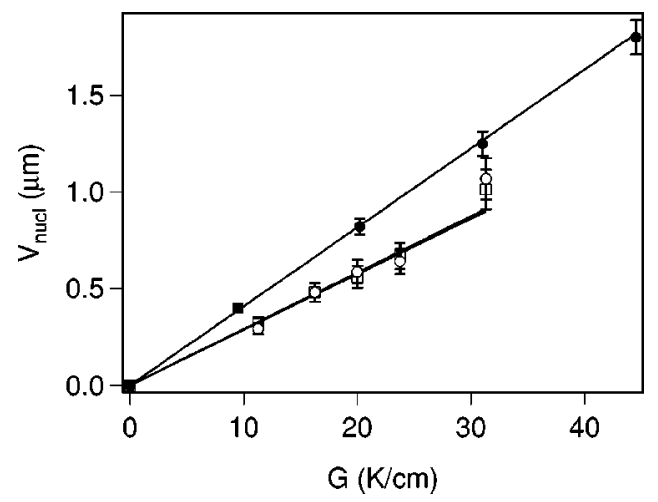

FIG. 7. By growing the phase that does not wet the glass plates we are able to observe nucleation of the new phase taking place before the cellular instability sets in. Empty symbols: velocity for nucleation of the isotropic phase upon melting the nematic phase in homeotropic samples. Thickness effects are not observed in the process. Samples of $b=10 \mu \mathrm{m}(\bigcirc)$ and $30 \mu \mathrm{m}(\square)$ are used. The slope corresponds to $D_{\perp} / \Delta T$, from which $D_{\perp}=(6.2 \pm 3)$ $\times 10^{-7} \mathrm{~cm}^{2} / \mathrm{s}$ is extracted. Full circles $(\bigcirc)$ : velocity for nucleation of the nematic phase upon freezing the isotropic phase in planar samples. A gap $b=30 \mu \mathrm{m}$ has been used. The slope corresponds to $D_{I} / \Delta T$, from which we obtain $D_{I}=(8.4 \pm 2) \times 10^{-7} \mathrm{~cm}^{2} / \mathrm{s}$.

diffusion coefficients. In order to measure $D_{\perp}$ and $D_{I}$ we have used the fact that, by driving our samples in the direction in which the phase that does not wet the glass plates is formed, nucleation of the new phase is observed before any cellular instability sets in. By melting the nematic phase in the homeotropic configuration, where this phase wets the plates, one finds a velocity for which the concentration profile in the nematic phase enters the coexistence region in the phase diagram. This is the condition for constitutional undercooling (or, rather, superheating, in the case where the nematic phase melts), and the critical velocity for the onset of nucleation can be expressed as $v_{\text {nucl }}=G D_{\perp} / \Delta T$. In order to accurately measure $v_{\text {nucl }}$, one must slowly increase the driving velocity, allowing for diffusion of the solute, until the constitutional superheating condition is achieved, which we take to be at the velocity for which the first isotropic bubble nucleates. Measuring $v_{\text {nucl }}$ for different $G$, and knowing $\Delta T$ we can estimate $D_{\perp}$ (see Fig. 7). We have verified that the value for $D_{\perp}$ obtained this way does not depend on the sample thickness. Only for thick samples (over $100 \mu \mathrm{m}$ thick) do we observe discrepancies, which are attributable to uncontrolled vertical temperature gradients, since our setup is designed to be used with thin samples. In order to test the validity of this method of measurement, we replaced I52 by a fluorescent dye. We found that the diffusion coefficient of the fluorescent dye obtained by measuring the velocity for constitutional superheating is in good agreement (within $10 \%$ ) with the one found by photobleaching (see [20] for a description of the experimental setup). Similarly, imposing a planar anchoring of the nematic molecules on the glass plates, and freezing the isotropic phase, we can estimate $D_{I}$ by measuring the velocity for constitutional undercooling. In this case, $v_{\text {nucl }}=G D_{I} / \Delta T$. Our estimations for the diffusion coefficients are $D_{I}=(8.4 \pm 2) \times 10^{-7} \mathrm{~cm}^{2} / \mathrm{s}$, and $D_{N}=(6.2$ $\pm 3) \times 10^{-7} \mathrm{~cm}^{2} / \mathrm{s}$. 
[1] P. Oswald, J. Bechhoefer, and A. Libchaber, Phys. Rev. Lett. 58, 2318 (1987).

[2] J. Bechhoefer, A. J. Simon, A. Libchaber, and P. Oswald, Phys. Rev. A 40, 2042 (1989).

[3] J. Bechhoefer, Ph.D. thesis, University of Chicago, 1988.

[4] W. W. Mullins and R. F. Sekerka, J. Appl. Phys. 34, 323 (1963).

[5] W. W. Mullins and R. F. Sekerka, J. Appl. Phys. 35, 444 (1964).

[6] J. S. Langer, Rev. Mod. Phys. 52, 1 (1980).

[7] L. Sallen, P. Oswald, and P. Sotta, J. Phys. II 7, 107 (1997).

[8] J. M. A. Figueiredo, M. B. L. Santos, L. O. Ladeira, and O. N. Mesquita, Phys. Rev. Lett. 71, 4397 (1993).

[9] J. Bechhoefer and S. A. Langer, Phys. Rev. E 51, 2356 (1995).

[10] C. Misbah and A. Valance, Phys. Rev. E 51, 1282 (1995).

[11] J. Ignés-Mullol, J. Baudry, L. Lejcek, and P. Oswald, Phys.
Rev. E 59, 568 (1999).

[12] B. Caroli, C. Caroli, and B. Roulet, J. Cryst. Growth 76, 31 (1986).

[13] S. de Cheveigné, C. Guthmann, and M. M. Lebrun, J. Phys. (France) 47, 2095 (1986).

[14] U. Finkenzeller, T. Geelhaar, G. Weber, and L. Pohl, Liq. Cryst. 5, 313 (1989).

[15] J. Cognard, Mol. Cryst. Liq. Cryst. Suppl. Ser. 78, 1 (1981).

[16] A. J. Simon and A. Libchaber, Phys. Rev. A 41, 7090 (1990).

[17] F. Melo and P. Oswald, Phys. Rev. Lett. 64, 1381 (1990).

[18] P. Oswald, J. Phys. II 1, 571 (1991).

[19] B. Caroli, C. Caroli, and B. Roulet, in Solids Far From Equilibrium, edited by C. Godrèche (Cambridge University Press, Cambridge, 1991), p. 218.

[20] J. Bechhoefer, J. C. Géminard, L. Bocquet, and P. Oswald, Phys. Rev. Lett. 79, 4922 (1997). 